\title{
Apport d'un modèle simplifié de simulation du bilan hydrique pour l'analyse de la satisfaction du besoin en eau de prairies irriguées des Alpes internes
}

\author{
D Trévisan ${ }^{1 *}, \mathrm{JM}$ Dorioz ${ }^{2}, \mathrm{Y}$ Pauthenet ${ }^{3}$ \\ 1 INRA, GIS Alpes du Nord, 75, avenue de Corzent, BP 511, F74203 Thonon-les-Bains; \\ ${ }^{2}$ INRA, 75, avenue de Corzent, BP 511, F74203 Thonon-les-Bains cedex, France; \\ ${ }^{3}$ Institut agricole régional, Région La Rochère 1/A, 111100 Aoste, Italie
}

(Reçu le 18 mars 1994 ; accepté le 9 janvier 1995)

\begin{abstract}
Résumé - Le recours à l'irrigation des prairies permanentes du val d'Aoste (Alpes italiennes) s'explique par la faible pluviosité estivale et la forte demande d'évapotranspiration. Pour étudier la satisfaction des besoins en eau de divers types de prairies irriguées, nous avons utilisé un modèle simplifié de calcul des variations de la teneur en eau du sol. II s'agit du module bilan hydrique de CREAMS (chemical, runoff, and erosion from agricultural management systems). Dans un premier temps, nous caractérisons la variabilité des différentes variables entrées dans le modèle : propriétés hydriques des sols, fréquences, dates et doses d'irrigation. Nous évaluons par ailleurs la valeur d'un paramètre de calage décrivant la répartition du système racinaire avec la profondeur. Dans un deuxième temps, nous simulons les variations de la teneur en eau du sol sur un réseau de 8 parcelles dont nous suivons l'humidité volumique et la production de matière sèche. Les calculs donnent des résultats comparables aux humidités observées et le rendement en matière sèche est corrélé significativement aux déficits hydriques moyens calculés. Le modèle ainsi validé nous permet de simuler le comportement hydrique de différentes parcelles représentatives de la variabilité régionale des sols et des pratiques d'irrigation.
\end{abstract}

Alpes / irrigation / comportement hydrique / modélisation

Summary - A simplified water-balance model for the analysis of the satisfaction of water requirements in irrigated inner Alps meadows. Irrigation of permanent meadows in Val d'Aosta (inner Italian Alps) is rendered necessary by low summer precipitations and by a high evapotranspiration demand. To study the satisfaction of the water requirement of vegetation, a simple model of water balance calculation was used. This is the 'hydrology' module of CREAMS (chemical, runoff, and erosion from agricultural management systems). We first characterized the variability of the different input variables: hydric properties of the soils, dates and frequencies of irrigation, and doses of water supplied. We also assessed the value of a parameter that relates root distribution with depth. We noticed a great variability of soil properties and irrigation practices. We then ran a simulation of water balance on 8 plots where soil moisture and dry-matter yield were monitored. The results of the calculations were comparable to the observed soil moisture values. Moreover, the dry-matter yields were correlated with the calculated mean water deficit. Simulations run with the model provide a framework adapted to the regional variability of soil properties and agricultural practices, which can be used to analyse interaction of the factors involved in the water balance.

\section{Alps / irrigation / hydric behaviour / modelling}

\footnotetext{
${ }^{*}$ Correspondance et tirés à part
} 


\section{INTRODUCTION}

Les typologies régionales de prairies permanentes apportent aux techniciens du développement agricole des outils permettant de raisonner les améliorations du système fourrager des exploitations (Hauwy et al, 1991). Dans le contexte des Alpes du Nord, la construction de ces typologies repose sur une analyse comparée des relations pratiques milieu-végétation dans un réseau de parcelles (100 à 200) représentatives de la diversité des conditions de milieu et des modalités d'exploitation des surfaces fourragères (Jeanin et al, 1991). Chaque parcelle de ce réseau fait l'objet d'enquêtes ou d'expertises relatives aux rythmes d'utilisation ainsi qu'au régime hydrique et trophique $\mathrm{du}$ sol. Le régime hydrique est apprécié par des critères globaux tels que profondeur du sol, taux de cailloux, texture et position topoclimatique. De tels critères sont opérationnels pour analyser l'effet des nombreux facteurs de différenciation des couverts végétaux (Fleury et al, 1985 ; Roumet et Fleury, 1992).

Une typologie des prairies est aujourd'hui en cours de réalisation dans le val d'Aoste, région où l'irrigation est systématique sur prairie jusqu'à $1500 \mathrm{~m}$ d'altitude, compte tenu du déficit hydrique estival typique des Alpes internes (Ozenda, 1981). Dans ces conditions, le diagnostic du régime hydrique doit tenir compte, en plus des critères habituels d'évaluation de la réserve utile, des interactions entre modalités d'irrigation et type de sol. L'objectif du travail présenté ici est de répondre à ce besoin de diagnostic, en analysant les conditions de la satisfaction en eau des prairies sur la base de simulations du bilan hydrique dans les diverses conditions du val d'Aoste. Le choix d'une approche par modélisation s'explique par la complexité et la diversité régionale des pratiques d'irrigation mises en œuvre dans le val d'Aoste, qui empêchent de mesurer exhaustivement leurs effets.

L'origine de l'irrigation remonte au Moyen Âge avec la construction d'un réseau de canaux acheminant l'eau de fonte des glaciers à des rigoles intraparcellaires que l'agriculteur met en fonction selon des usages traditionnels (Janin, 1967). Ces dernières années, ce système d'irrigation empirique par ruissellement a été partiellement remplacé par un réseau d'irrigation par aspersion (Roumet et al, 1992). La gestion des réseaux d'irrigation est en fait très différente selon qu'il s'agit de ruissellement ou d'aspersion. Dans le cas de l'irrigation par aspersion, les doses et fréquences des apports sont établies à partir de paramètres très globaux tels que rendement potentiel de la végétation ou réserve utile standardisée (Bernard, 1990). Dans le cas du ruissellement, le partage des tours d'eau s'effectue selon «l'égance», règlement ancien qui fixe, sur la base de critères relatifs à la propriété des parcelles, les périodes durant lesquelles le terrain a droit à l'eau (Janin, 1967). L'agriculteur décide ensuite de l'utilisation de son tour d'eau et contrôle l'irrigation en faisant déborder les rus intra-parcellaires. On est donc loin en val d'Aoste des pratiques d'irrigation des zones de grande culture, prenant en compte l'optimisation des réseaux (ITCF, 1993), le rendement des cultures (Voltz et Rambal, 1987) ou l'incidence sur la salinité des sols (Durand, 1983 ; Meddahi et al, 1993), même s'il existe très localement des références pour optimiser l'irrigation de surfaces fourragères d'altitude (Blondon et al, 1987).

Pour être opérationnelle, la modélisation du bilan hydrique doit rendre compte de la diversité des conditions d'alimentation en eau des plantes à l'échelle régionale (100 000 ha). Ceci suppose de faire appel à des modèles utilisant peu de paramètres. Ces derniers doivent de plus être aisément accessibles, soit à partir d'enquêtes sur les pratiques, soit à partir de descriptions de terrain sur les conditions pédologiques. De telles contraintes excluent l'usage de modèles déterministes décrivant les processus physiques de transfert de l'eau (Gras, 1988) ou stochastiques, prenant en compte la variabilité spatiale des paramètres d'état (Vauclin, 1990).

La solution adoptée consiste à utiliser un modèle empirique, à l'adapter en vue de décrire le stress hydrique dans les différents types de parcelles de la région d'étude. Les simulations réalisées à partir de ce modèle sont utilisées pour repérer des pratiques ou des situations de milieu où la disponibilité en eau risque d'être limitante pour la croissance végétale.

\section{MÉTHODOLOGIE}

\section{Vue d'ensemble sur le modèle utilisé}

Il existe plusieurs méthodes de calcul du bilan hydrique, présentant une complexité plus ou moins grande selon qu'elles prennent en compte, explicitement ou non, un certain nombre de processus physiques (Choisnel, 1992). Kauark Leite (1990) en propose une classification et montre l'intérêt des modèles conceptuels. Les modèles CREAMS (Knisel, 1980), 
PROBE (Choppart et Vauclin, 1990) ou CNS (Haith et al, 1984) s'apparentent à de tels modèles puisqu'ils ont recours à des représentations simplifiées des variations des teneurs en eau du sol. Notre choix s'est porté sur CREAMS en raison des nombreuses applications dont il a fait l'objet (Kauark Leite, 1990) et du nombre restreint de paramètres nécessaires aux calculs.

CREAMS se décompose en plusieurs sousmodèles indépendants de simulation du bilan hydrique des sols d'un bassin versant et de transfert de polluants. Nous ne nous intéressons ici qu'au modèle de CREAMS de calcul des variations journalières des teneurs en eau des sols.

La réponse à la demande d'évapotranspiration est décrite dans ce module de la façon suivante :

- i) un partage de l'ETP entre évaporation et transpiration. Lorsque l'indice foliaire (rapport entre surface des feuilles et surface du sol) est $>3$, on considère que l'évaporation est nulle ;

- ii) une fonction définissant la transpiration réelle TR. Elle est maximale et égale à ETP lorsque I'humidité volumique du sol n'est pas limitante, c'est-à-dire lorsqu'elle est supérieure au quart de la réserve utile. Inversement et pour tenir compte des phénomènes de régulation stomatique, lorsque l'humidité volumique est inférieure à cette limite, on a :

$$
T R=E T P \frac{S M}{0,25 R U}
$$

avec $S M$ le stock d'eau libre (humidité volumique du sol - humidité volumique au point de flétrissement) et $R U$ la réserve utile ;

- iii) une fonction définissant la distribution de TR dans les couches de sol (Williams et Haan, 1978):

$$
u=u_{0} \mathrm{e}^{-\alpha \frac{z}{R}}
$$

avec $u$ le taux d'utilisation de l'eau par les plantes à une profondeur $z$ donnée, $u_{0}$ le taux d'utilisation à la surface du sol, $\mathrm{R}$ la profondeur racinaire et $\alpha$ un paramètre décrivant la répartition des racines avec la profondeur ( $\alpha$ est d'autant plus élevé que la densité racinaire est plus forte en surface).

Dans le cas d'une journée pluvieuse ou d'une irrigation, les entrées d'eau dans le sol sont définies par $P+I-E T P$ (avec $P$ la pluviométrie journalière et $I$ la lame d'eau apportée par irrigation). Si cette grandeur est négative, la demande d'évaporation est répartie dans le profil de sol selon [2]. Dans le cas contraire, la lame d'eau apportée est ajoutée à I'humidité volumique initiale. Si l'humidité volumique résultante est supérieure à la capacité au champ, c'est cette dernière qui est retenue comme humidité volumique finale, le trop plein étant reporté dans un réservoir sousjacent. Enfin, les échanges d'eau liés à des gradients de teneur en eau ne sont pas pris en compte dans le modèle.

\section{Dispositif expérimental}

La mise en œuvre d'un tel modèle nécessite la connaissance de variables d'entrée (ETP et précipitations journalières), de paramètres relatifs aux caractéristiques édaphiques (réserve utile) et végétales (profondeur d'enracinement, indice foliaire), ainsi que du coefficient $\alpha$. Le dispositif expérimental que nous avons mis en place pour connaître ces différentes variables est récapitulé au tableau I. II comprend des mesures de teneur en eau du sol et de production de matière sèche et des enquêtes portant sur les modalités et les pratiques d'irrigation. Les différentes valeurs de teneur en eau sont obtenues par méthode gravimétrique, avec pour chaque valeur et chaque profondeur de mesure, 3 prélèvements de terre à la tarière.

\section{Caractéristiques édaphiques}

Une analyse multidimensionnelle des paramètres édaphiques recueillis lors de sondages pédologiques (nombre d'horizons, texture, profondeur, taux de cailloux et profondeur d'enracinement) a permis d'analyser la différenciation de la couverture pédologique et de dresser une typologie des sols (Trévisan et al, 1992). Les sols sont tous développés sur formations superficielles graveleuses telles que cônes de déjection, moraines ou colluviums. Sept types de sol ont été distingués, selon l'état du complexe adsorbant, la texture, la profondeur et la charge en cailloux (tableau II). Ces différents types peuvent être regroupés en classes de mêmes propriétés hydriques : une classe C1 regroupe les types de sols superficiels et caillouteux (type 1 et type 5) ; une classe $C 2$ les types moyens $2,4,6$; et C3 les types les plus profonds 3 et 7 .

Chaque classe de sol a fait l'objet d'une série de mesures destinées à caractériser la réserve en eau du sol (cf tableau I, ligne 1) : masse volumique (méthode au sable, Baize, 1988), taux de cailloux (Rochas, 1991) et capacité au champ (obtenue par mesure de l'humidité $48 \mathrm{~h}$ après une irrigation par submersion, Boulaine, 1978).

\section{Indice foliaire}

L'indice foliaire est corrélé à la hauteur de l'herbe et au stade de développement de la prairie. D'après Williams et al (1980), il est supérieur à 3 dans le cas des prairies en période estivale. Selon Duru (1989) cette limite est dépassée dès que l'herbe des prairies permanentes atteint 15 à $20 \mathrm{~cm}$, selon la fertilisation. En montagne, la croissance de l'herbe est très rapide au printemps (Fleury et al, 1989). Ainsi dans le val d'Aoste, la hauteur de l'herbe à la mi-avril, mesurée sur des parcelles expérimentales représentatives, est de $24 \mathrm{~cm} \pm 4$ (tableau I, ligne 4). Les repousses après fauche (mi-mai à mi-juin) sont par ailleurs très rapides, compte tenu de l'irrigation et des conditions thermiques, et les hauteurs d'herbe sont de $23 \mathrm{~cm} \pm 5,15$ j après la coupe. Finalement, durant les 9 dixièmes de 
Tableau I. Dispositif expérimental.

Objectif détaillé

Caractérisation de la
réserve utile du sol

III

Mesure de teneur en eau consécutive aux irrigations
Méthodes

Détermination de la masse volumique

(méthode au sable)

Détermination du taux de cailloux (pesée du refus de tamisage à $2 \mathrm{~mm}$ )

Détermination de la capacité au champ (mesure de teneur en eau $48 \mathrm{~h}$ après saturation sous irrigation par submersion)

Mesure de teneur en eau à 2 profondeurs différentes (prélèvement de terre $2,3,5,7,10$ et $15 \mathrm{j}$ après irrigation)

Mesures de teneur en eau la veille et $24 \mathrm{~h}$ après irrigation
Nombre de parcelles ou de répétitions

3 parcelles représentatives de la classe de sol

$n=18$ à 32 selon la classe de sol

$n=2$ à 9 selon la classe de sol

3 parcelles représentatives de la classe de sol. Deux séquences de dessication par parcelle

17 parcelles représentatives de la classe de sol et des modalités d'irrigation (aspersion ou ruissellement, fréquence d'apport d'eau de $7,10,15,20 \mathrm{j}$ selon les cas)

- Mesures de teneur en eau tous les 15 j à 30 et $50 \mathrm{~cm}$ - Mesure de la production de matière sèche (coupe à $2 \mathrm{~cm}$ sur $1^{\mathrm{er}}$ et $2^{\mathrm{e}}$ cycle) et des hauteurs de végétation en place

(50 mesures sur un transect de $5 \mathrm{~m}$ )

- Mesure de l'angle de la ligne d'horizon selon 8 directions cardinales
8 parcelles représentatives de la classe de sol et des pratiques de fertilisation comportement hydrique prédit au rendement en matière sèche

Classe 2

Profondeur moyenne

et

taux de cailloux moyen

Type 2

Limono-sableux

Type 4

Limono-sableux

Type 6

Limono-sableux
Classe 3

Profondeur forte

et

taux de cailloux faible

Type 3

Limono-sableux
Sols calciques

Sols décarbonatés
Type 5

Sablo-limoneux
Type 7

Limoneux 
la période d'étude et de simulation des variations des teneurs en eau du sol (début avril à fin août), on peut considérer que l'indice foliaire est supérieur à 3 .

\section{Coefficient $\alpha$}

La valeur du coefficient $\alpha$ peut être définie expérimentalement i) si on considère que l'évaporation à partir du sol lui-même est nulle en raison de la forte couverture végétale pendant la saison estivale ; ii) si on néglige les remontées capillaires, ce qui est acceptable dans nos conditions particulières de texture sableuse de l'altérite et d'absence de nappe. Par conséquent, en dehors des périodes de pluie, les variations d'humidité volumique du sol sont du seul ressort de la transpiration végétale et on peut écrire :

$$
\left(h_{0}-h_{\mathrm{i}}\right)_{\mathrm{z}}=T R_{z}
$$

avec $h_{0}$ l'humidité volumique initiale, $h_{\mathrm{i}}$ l'humidité volumique au jour i et $T R_{Z}$ la perte d'eau dans la couche $z$.

En introduisant [2], on obtient:

$$
\left(h_{0}-h_{\mathrm{i}}\right)_{0-z 1}=T R_{0-z 1}=\frac{\mathrm{u}_{0}}{\alpha}\left(1-\mathrm{e}^{-\alpha \frac{z 1}{\mathrm{R}}}\right)
$$

et

$$
\left(h_{0}-h_{\mathrm{i}}\right)_{z 1-z 2}=T R_{z 1-z 2}=\frac{\mathrm{u}_{0}}{\alpha}\left(\mathrm{e}^{-\alpha \frac{z 1}{\mathrm{R}}}-\mathrm{e}^{-\alpha \frac{z 2}{\mathrm{R}}}\right)[4]
$$

Et, en combinant [3] et [4] :

$$
\left(h_{0}-h_{i}\right)_{z 1-z 2}=\left(h_{0}-h_{i}\right)_{0-z 1} \frac{e^{-\alpha \frac{z 1}{R}}-e^{-\alpha \frac{z 2}{R}}}{1-e^{-\alpha \frac{z 1}{R}}}
$$

Connaissant $\left(h_{0}-h_{\mathrm{i}}\right)$ à 2 profondeurs différentes $z_{1}$ et $z_{2}$, l'équation [5] permet de définir expérimentalement $\alpha$.

Nous avons ainsi mis en place, sur des parcelles représentatives des 3 classes de sol, un suivi des teneurs en eau $\left(h_{0}-h_{i}\right)$ à $z_{1}=30$ et $z_{2}=50 \mathrm{~cm}$ lors de 2 séquences de dessiccation du sol (cf tableau I, ligne 2).

\section{Teneurs en eau après irrigation}

L'adaptation du modèle à nos conditions d'irrigation nécessite un développement particulier. En effet, avec l'irrigation par ruissellement, les doses d'apport d'eau sont impossibles à quantifier, compte tenu de la multitude de facteurs pouvant influencer les volumes d'apport tels que micro-relief, dimension et entretien du réseau de canaux, pertes lors du parcours de l'eau (Bernard, 1990). Nous avons donc eu recours à une évaluation indirecte de ces doses d'apport, en mesu- rant les variations des teneurs en eau consécutives aux irrigations.

Ces mesures sont effectuées la veille et $24 \mathrm{~h}$ après irrigation, sur différentes parcelles représentatives des sols et des pratiques d'irrigation (tableau I, ligne 3). Les pratiques sont connues à partir d'une enquête auprès des usagers portant sur 102 sites. Les informations recueillies servent à repérer les valeurs moyennes et les extrêmes en termes de calendrier et de fréquence d'apport d'eau, ainsi que des variations dues au mode d'irrigation (saison plus longue et apports plus fréquents sous aspersion, cf tableau III).

\section{Suivis d'humidité volumique}

Pour analyser la fiabilité des simulations, les calculs ont été confrontés à des suivis d'humidité volumique de 8 parcelles test et à des mesures de leur production de matière sèche (tableau I, ligne 4). Les modalités de gestion de I'herbe de ces parcelles (tableau IV) sont représentatives des pratiques d'irrigation et de fertilisation du val d'Aoste (Roumet et al, 1992). Pour chaque parcelle, l'évapotranspiration potentielle journalière est calculée d'après la formule de Penman. Le calcul de l'ETP Penman tient compte de l'énergie solaire qui est calculée à l'aide du programme FLASH (Durand et Legros, 1981 ; Legros et al, 1986) pour tenir compte de l'exposition du site et des effets d'occultation par le relief. Le programme requiert pour chaque situation les valeurs de l'angle de la ligne d'horizon : elles sont mesurées avec un clinomètre, selon 8 directions cardinales.

\section{RÉSULTATS ET DISCUSSION}

Un premier ensemble de résultats concerne la caractérisation de la réserve en eau des sols et leur comportement durant les phases de réhumectation et de dessiccation. II nous permet de définir les paramètres et variables requis par le modèle.

\section{Évaluation des paramètres du modèle}

\section{Caractérisation de la réserve en eau des sols du val d'Aoste}

La texture des sols est largement dominée par les sables. II s'agit là de conditions radicalement différentes de celles observées dans les Alpes externes où les textures sont en majorité limonoargileuses (Dorioz et Van Oort, 1991 ; Legros, 1986). La dominance sableuse des sols des Alpes internes pourrait être liée aux conditions climatiques (Legros, 1984). 
Tableau III. Fréquences relatives de début de saison d'irrigation, de fin de saison d'irrigation et de fréquence d'irrigation en val d'Aoste.

\begin{tabular}{|c|c|c|c|c|}
\hline Mode & Début d'irrigation & Fin d'irrigation & Durée irrigation & Fréquence \\
\hline \multirow{6}{*}{ Aspersion } & Début avril : 25 & & $\begin{array}{l}105 \mathrm{j}: 0 \\
120 \mathrm{j}: 10\end{array}$ & \\
\hline & Mi-avril : 20 & Début septembre : 0 & $135 \mathrm{j}: 15$ & $7 \mathrm{j}: 30$ \\
\hline & Fin avril : 30 & Mi-septembre : 50 & $150 j: 5$ & $10 j: 20$ \\
\hline & Début mai : 15 & Fin septembre : 50 & $165 \mathrm{j}: 35$ & $15 \mathrm{j}: 50$ \\
\hline & Mi-mai : 10 & & $180 \mathrm{j}: 25$ & $20 \mathrm{j}: 0$ \\
\hline & Fin mai : 0 & & $195 \mathrm{j}: 10$ & \\
\hline \multirow{6}{*}{ Ruissellement } & Début avril : 20 & & $\begin{array}{l}105 \mathrm{j}: 5 \\
120 \mathrm{j}: 10\end{array}$ & \\
\hline & Mi-avril : 10 & Mi-septembre : 40 & $135 \mathrm{j}: 40$ & $7 \mathrm{j}: 0$ \\
\hline & Fin avril : 10 & Mi-septembre : 40 & $150 j: 5$ & $10 j: 5$ \\
\hline & Début mai : 30 & Fin septembre: 40 & $165 j$ : 20 & $15 j: 65$ \\
\hline & Mi-mai : 15 & & $180 \mathrm{j}: 5$ & $20 j: 30$ \\
\hline & Fin mai : 15 & & $195 \mathrm{j}: 15$ & \\
\hline
\end{tabular}

Tableau IV. Caractéristiques des parcelles de suivi d'humidité et de production de matière sèche.

\begin{tabular}{|c|c|c|c|c|c|}
\hline $\begin{array}{l}\text { Fertilisation } \\
\text { (UN/ha) }\end{array}$ & Classe de sol & $\begin{array}{c}\text { Modalité } \\
\text { d'irrigation }\end{array}$ & $\begin{array}{l}\text { Fréquence } \\
\text { d'irrigation }\end{array}$ & $\begin{array}{c}\text { Début } \\
\text { d'irrigation }\end{array}$ & $\begin{array}{c}\text { Nombre } \\
\text { d'irrigation }\end{array}$ \\
\hline 75 & C3 & Aspersion & $15 \mathrm{j}$ & Mi-juin & 3 \\
\hline 180 & C3 & Ruissellement & $15 j$ & Mi-mai & 6 \\
\hline 85 & $\mathrm{C} 2$ & Ruissellement & $10 \mathrm{j}$ & Fin mai & 5 \\
\hline 154 & C3 & Ruissellement & $21 \mathrm{j}$ & Début mai & 3 \\
\hline 50 & $\mathrm{C} 1$ & Ruissellement & $15 \mathrm{j}$ & Fin mai & 7 \\
\hline 165 & $\mathrm{C} 2$ & Ruissellement & $15 j$ & Début mai & 5 \\
\hline 192 & C3 & Ruissellement & $15 j$ & Mi-avril & 6 \\
\hline 200 & $\mathrm{C}_{1}$ & Aspersion & $15 j$ & Début juillet & 4 \\
\hline
\end{tabular}

UN : unité d'azote

II existe cependant une diversité de texture et de réserve hydrique selon les classes de sol (tableau V). Lorsque l'on passe de la classe $\mathrm{C} 1$ à $\mathrm{C} 3$, les textures varient des sables limoneux aux limons moyens sableux, les taux de cailloux de 30 à $5 \%$. Ceci se traduit par des réserves hydriques de plus en plus fortes, comme le confirment les valeurs d'humidité pondérale mesurées après $48 \mathrm{~h}$ de ressuyage.

\section{Variations des teneurs en eau en phase de dessiccation des sols et évaluation du coefficient $\alpha$}

Les pertes d'humidité volumique $h_{0}-h_{\mathrm{i}}$ observées après irrigation sont liées à la demande d'évapotranspiration. Elles sont également influencées par différentes caractéristiques édaphiques (fig 1).

- i) la classe de sol : les cinétiques de dessiccation sont plus rapides lorsque l'on passe de la classe $\mathrm{C} 3$ à $\mathrm{C} 2$ puis $\mathrm{C} 1$. Ceci est en accord avec les résultats de Puech (1969) et Gras (1988) qui montrent des dessiccations plus rapides pour le pôle sableux ;

- ii) la profondeur de sol ; avec des cinétiques plus rapides en surface qu'en profondeur.

Les comportements hydriques pendant la phase de dessiccation sont ainsi bien distincts selon la classe de sol.

Les couples de données $\left(h_{0}-h_{\mathrm{i}}\right)$ à 30 et 50 $\mathrm{cm}$ nous permettent d'appliquer l'équation [5], 
Tableau V. Caractéristiques analytiques des différentes unités de sol.

\begin{tabular}{|c|c|c|c|c|}
\hline $\begin{array}{c}\text { Texture } \\
\text { (Surface/profondeur) }\end{array}$ & $\begin{array}{l}\text { Densité } \\
\text { apparente }\end{array}$ & $\begin{array}{c}\text { Taux de } \\
\text { cailloux }(\%)\end{array}$ & $\begin{array}{l}\text { Humidité } 48 \mathrm{~h} \\
\text { après irrigation }\end{array}$ & $\begin{array}{l}\text { Profondeur } \\
\text { utile }(\mathrm{cm})\end{array}$ \\
\hline SL/SL & $1,3-1,45$ & $34,1(n=18)$ & $\begin{array}{l}14,3(30 \mathrm{~cm}, n=4) \\
11,5(50 \mathrm{~cm}, n=4)\end{array}$ & $40-80$ \\
\hline LS/SL & $1,3-1,4$ & $21,3(n=32)$ & $\begin{array}{l}19,5(30 \mathrm{~cm}, n=2) \\
14,5(50 \mathrm{~cm}, n=2)\end{array}$ & $80-120$ \\
\hline LS/LS & $1,3-1,4$ & $10,2(n=20)$ & $\begin{array}{l}31,9(30 \mathrm{~cm}, n=9) \\
30,1(50 \mathrm{~cm}, n=6)\end{array}$ & $100-120$ \\
\hline
\end{tabular}

CI
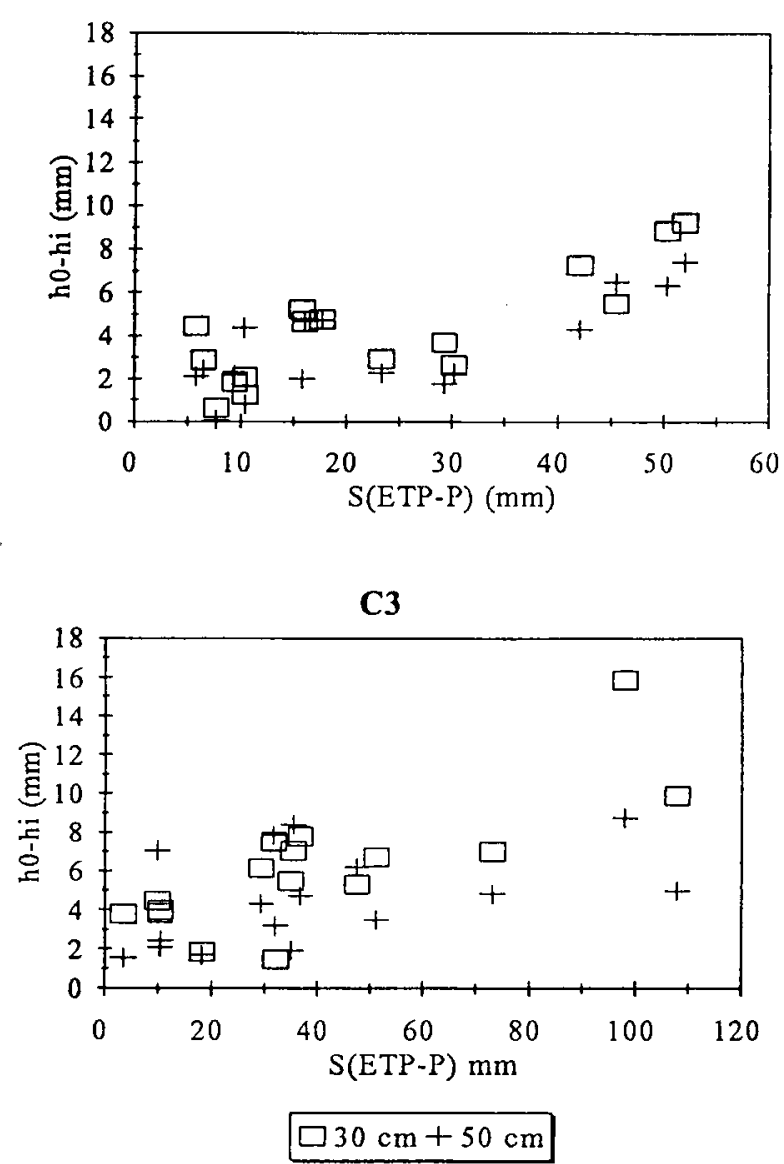

avec $R$ considéré comme égal à 80,100 et 120 $\mathrm{cm}$ pour respectivement les classes $\mathrm{C} 1, \mathrm{C} 2$ et C3, valeurs évaluées lors de l'établissement de la typologie des sols. Les valeurs d' $\alpha$ ajustées sont similaires et faibles pour $\mathrm{C} 1$ et $\mathrm{C} 2$, alors que, dans le cas des sols plus limoneux C3, $\alpha$ ajusté est comparable à $\alpha=3,065$, valeur employée par Kauark Leite (1990) (fig 2). Les faibles valeurs d' $\alpha$ dans le cas des classes $C 1$ et C2 signifient probablement qu'il existe une forte contribution des couches profondes du sol à l'alimentation racinaire. Inversement, dans le cas
C2

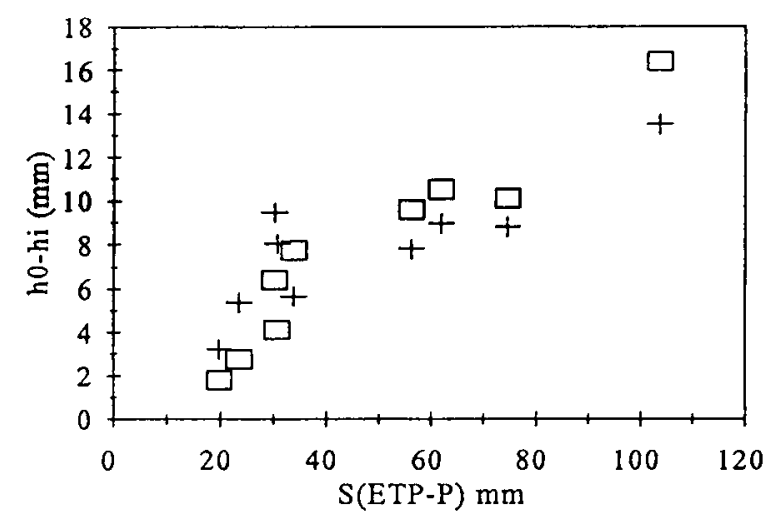

Fig 1. Relations entre pertes d'humidité pondérale (ho-hi) mesurée à 30 et $50 \mathrm{~cm}$ et demande de déficit climatique $\Sigma$ $(E T P-P)$ dans 3 sols représentatifs des unités de sols sous prairies $(\mathrm{C} 1, \mathrm{C} 2, \mathrm{C} 3)$ (ho: humidité volumique $24 \mathrm{~h}$ après irrigation; hi: humidité volumique du jour $i$ ).

des sols moins sableux et faiblement caillouteux, $\alpha$ est plus fort, ce qui traduit une répartition plus superficielle des racines et suggère une alimentation hydrique mettant plus à contribution les horizons de surface.

\section{Teneur en eau du sol après irrigation}

On constate qu'il existe une relation linéaire liant les humidités pondérales avant irrigation $(J-1)$ à celles après irrigation $(J+1)$ (fig 3$)$. Les teneurs 
$\mathrm{Cl}$

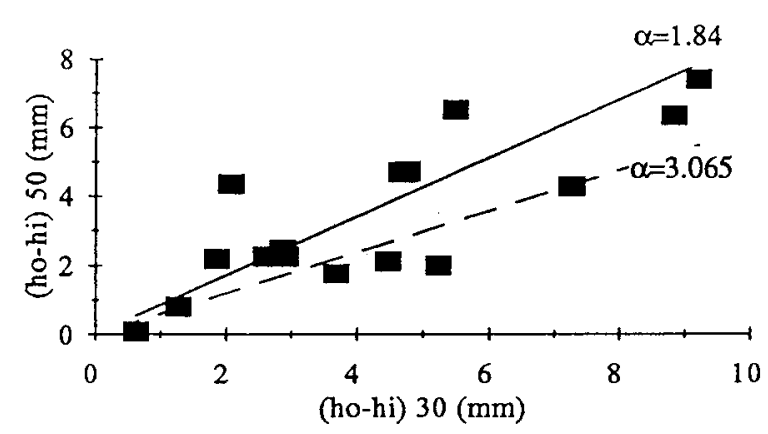

c3

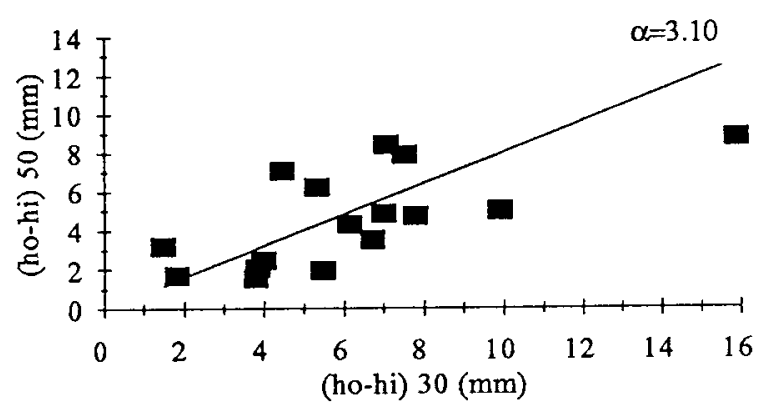

en eau après irrigation $(J+1)$ sont proches de la capacité au champ des différentes classes de sol, avec des valeurs de $\mathrm{J}+1$ centrées sur $15 \%$ pour les sols de classe $\mathrm{C} 1$ et de $30 \%$ pour les sols plus profonds C3.

Les valeurs d'humidité pondérale avant l'irrigation dépendent également en grande partie des conditions édaphiques. Pour les sols de la classe $C 1$, elles sont comprises entre 5 et $10 \%$, ce qui est proche des valeurs correspondant au point
$\mathrm{C} 2$

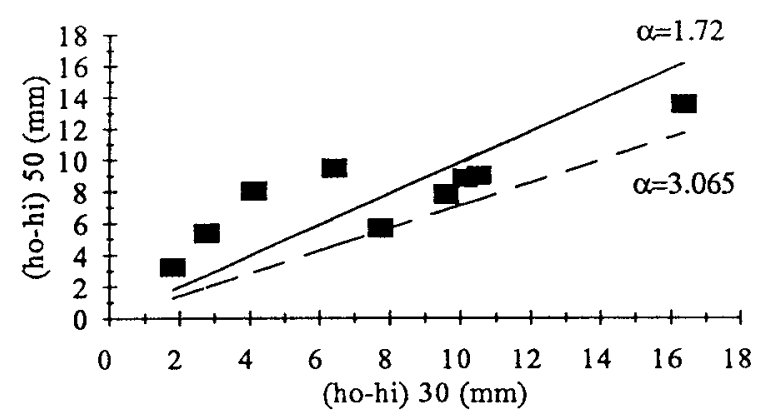

Fig 2. Pertes d'humidité pondérale à 30 et $50 \mathrm{~cm}$ et ajustement du coefficient $\alpha$. En trait continu : ajustement du coefficient $\alpha$ aux données. En trait pontillé, valeur proposée par Kauark Leite (1990).

de flétrissement des sols sableux ( $7 \%$ selon Gras, 1988). Inversement, dans le cas des sols $\mathrm{C} 3$, les humidités pondérales avant irrigation sont nettement supérieures à celles du point de flétrissement retenu pour ce type de sol limoneux (15\% selon Gras, 1988). Ces faits montrent que, globalement, les pratiques ne prennent pas en compte les propriétés hydriques des sols.

Dans la mesure où les caractéristiques édaphiques expliquent une partie de la variabilité des

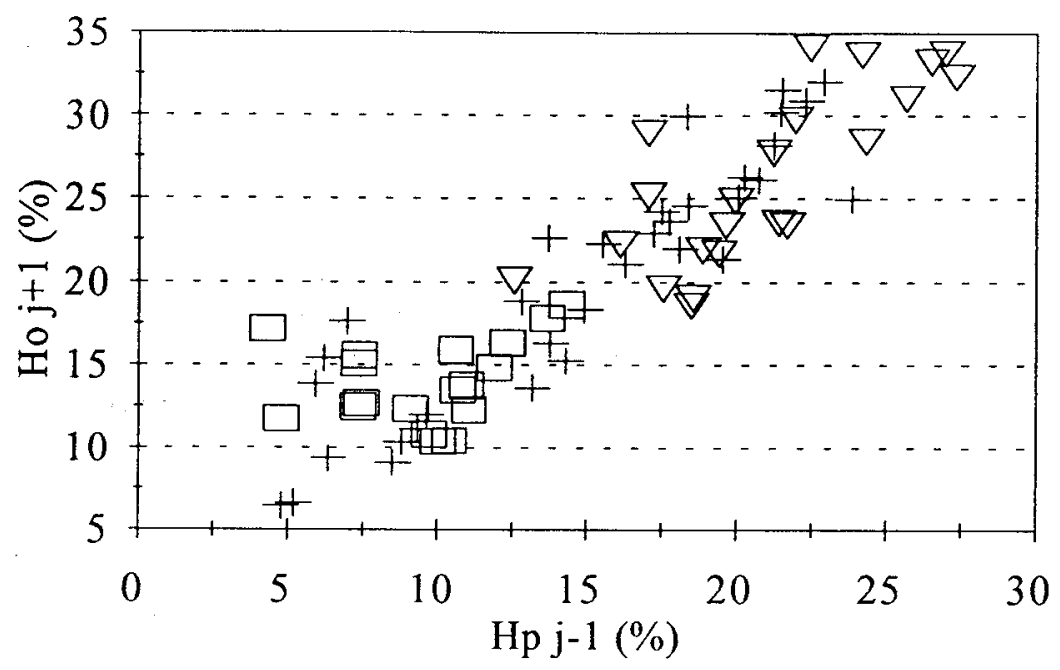

sols $\mathrm{C} 1+$ sols $\mathrm{C} 2 \nabla$ sols $\mathrm{C} 3$

Fig 3. Humidités pondérales avant $(H p j-1)$ et après $(H p J+1)$ irrigation. 
valeurs obtenues, les classes de sol peuvent servir de premier critère de classement de la teneur en eau après irrigation. Nous avons donc tenté ensuite de comprendre pour chaque classe de sol la dispersion des valeurs de $\mathrm{J}+1$ (fig 4). On constate que, quelle que soit la profondeur, la dispersion des résultats pour les classes de sol $\mathrm{C} 1$ et $\mathrm{C} 2$ est toujours plus forte sous aspersion que sous ruissellement. Sous aspersion, de nombreux facteurs tels que pression d'amenée d'eau, diamètre des buses, vent, fréquence ou durée d'irrigation peuvent diversifier les teneurs en eau. Ainsi dans le cas $\mathrm{C} 1$ et $\mathrm{C} 2$, la fréquence d'aspersion explique une partie de la dispersion. Pour les sols $\mathrm{C} 3$ en revanche. c'est la profondeur de

\section{C1}

Nbre d'observations

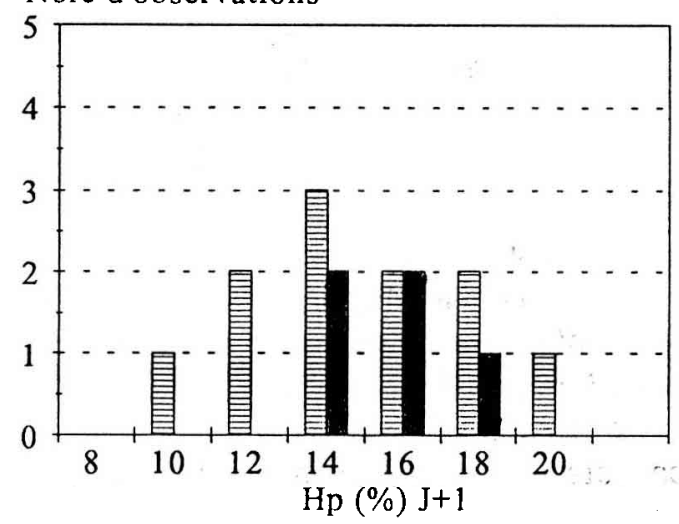

目aspersion $(30$ et $50 \mathrm{~cm}$ )

ruissellement (30 et $50 \mathrm{~cm}$ )

Nbre d'observations

C3

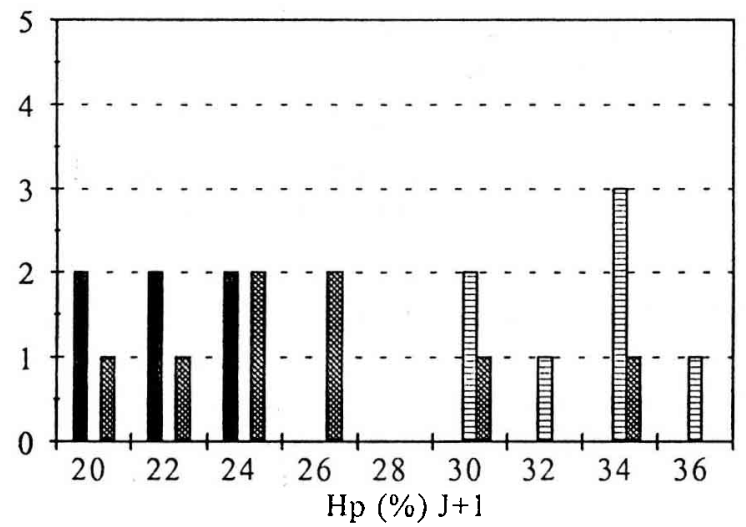

aspersion 30 et $50 \mathrm{~cm}$

国ruissellement $30 \mathrm{~cm}$

ruissellement $50 \mathrm{~cm}$ sol qui est à l'origine de la variabilité des teneurs $\mathrm{J}+1$. Ceci s'explique si on considère que, sous ruissellement, l'agriculteur n'arrête généralement l'irrigation qu'après la submersion des parcelles. Dans le cas des sols C3, moins filtrants, on peut penser que, $24 \mathrm{~h}$ après la submersion, la redistribution de l'eau excédentaire de l'horizon superficiel n'est pas complète, ce qui explique les différences entre 30 et $50 \mathrm{~cm}$.

Sur ces bases, il est possible de proposer une valeur d'humidité pondérale moyenne $H i$ après irrigation, pour chacune des différentes classes de sols et de modalités d'irrigation (tableau VI).

Connaissant les valeurs caractéristiques de la réserve en eau, le coefficient $\alpha$ et les teneurs $\mathrm{Hi}$,

\section{C2}

Nbre d'observations

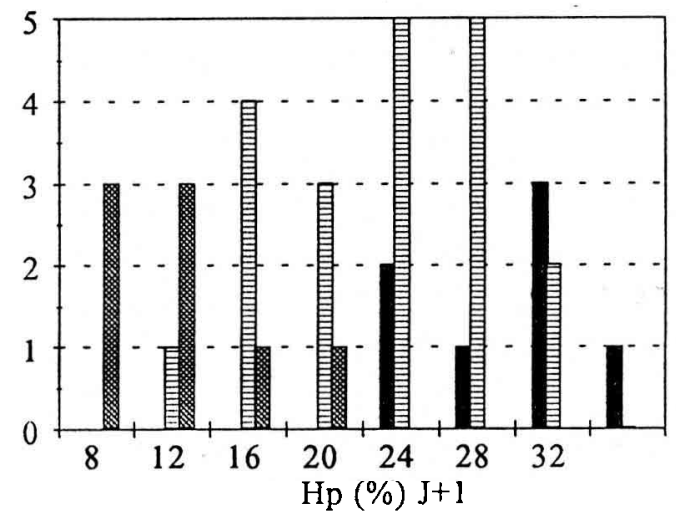

ruissellement (30 et $50 \mathrm{~cm}$ )

国aspersion de fréquence 7 à $10 \mathrm{j}(30$ et $50 \mathrm{~cm}$ )

aspersion de fréquence supérieure à $10 \mathrm{j}$ (30 et $50 \mathrm{~cm}$ )
Fig 4. Distribution des humidités pondérales à 30 et $50 \mathrm{~cm}$ après irrigation dans les différentes unités de sol. 
Tableau VI. Humidités pondérales moyennes Hi après irrigation selon le type de sol et les modalités d'irrigation.

Aspersion
Ruissellement
nous pouvons simuler les variations des teneurs
en eau en croisant tous les cas rencontrés sur le
terrain pour dégager des indicateurs sur la satis-
faction de la demande en eau des plantes. Ceci
suppose d'abord de valider le modèle.

\section{Mise en cuvre et validation du modèle}

Dans le but d'analyser la fiabilité du modèle, les calculs ont été effectués sur 8 parcelles test ( $c f$ tableau IV), puis confrontés à des valeurs d'humidité mesurées et aux productions de matière sèche des couverts végétaux.

\section{Simulation des variations des teneurs en eau}

Nous avons simulé les variations d'humidité volumique du sol, en considérant 2 réservoirs : un réservoir superficiel (de 0 à $30 \mathrm{~cm}$ ) et un réservoir sous-jacent de 30 à $50 \mathrm{~cm}$. La limite à $50 \mathrm{~cm}$ est fixée pour des raisons pratiques, les contrôles de teneur en eau étant très difficiles à réaliser plus profondément dans les sols caillouteux.

Le calcul s'effectue de fin avril à fin juillet, période durant laquelle on peut considérer la profondeur d'enracinement $R$ comme constante, comme suggéré par West et al (1989) qui montrent que les longueurs d'enracinement se stabilisent au bout de 2 années succédant à l'implantation d'une prairie. Au vu d'autres travaux, cette hypothèse semble également fondée : au cours d'une saison, le système racinaire est moins sensible aux fertilisations que la partie aérienne (Toth, 1989). De même, ce n'est qu'à la troisième coupe annuelle qu'une baisse de la biomasse racinaire est observable (Lyszczarz, 1989). En revanche, les enracinements sont très fortement liés aux contraintes hydriques, avec une augmentation de l'exploration racinaire dans des conditions de dessiccation des horizons de surface (Henin et al, 1969). Dans notre contexte, la nature graveleuse des horizons profonds limite

Sols $C 2$
$>10 \mathrm{j}: 0,12$
$>10 \mathrm{j}: 0,22$

0,28
Sols $C 3$

0,22

$30 \mathrm{~cm}: 0,34$

$50 \mathrm{~cm}: 0,26$ sans doute de telles adaptations et nous avons en conséquence considéré que la profondeur d'enracinement de la période estivale est constante et correspond à la profondeur utile du sol, c'est-à-dire dans nos conditions, à la profondeur d'apparition de l'horizon d'altération (respectivement $80,100,120 \mathrm{~cm}$ pour $\mathrm{C} 1, \mathrm{C} 2, \mathrm{C} 3$ ). D'après [2], on peut alors calculer que la contribution de la couche $[0-50 \mathrm{~cm}$ ) à la demande d'évapotranspiration est de $65,70,80 \%$ pour respectivement les classes de sol C3, C2 et C1.

L'initialisation du modèle est effectuée début mai avec une valeur d'humidité volumique mesurée. Les calculs sont poursuivis ensuite jusqu'à fin juillet. Après chaque irrigation, l'humidité volumique du sol est amenée à la valeur moyenne $\mathrm{Hi}$, caractéristique de la modalité d'irrigation (tableau V1). Lorsque cette humidité volumique est supérieure à la capacité au champ, c'est cette dernière qui est retenue.

Le résultat des calculs (fig 5) montre que les valeurs d'humidité volumique $H V$ calculées suivent assez bien celles observées ( $H V$ calculée $=$ $0,84 H v$ observée $+2,7 ; r^{2}=0,73, n=181$ ). Le décalage résiduel entre prédiction et observation est lié à l'imprécision de certaines informations comme les dates d'irrigation du réseau de parcelles de suivi d'humidité volumique qui sont obtenues par enquêtes auprès des exploitants.

Les calculs sont, en outre, largement dépendants des teneurs en eau Hi après irrigation, comme le montre l'analyse de sensibilité des paramètres d'entrée (fig 6). En faisant varier les inputs dans une gamme de valeurs qui reste plausible par rapport à la réalité du terrain, il apparaît que le rang de sensibilité est par ordre décroissant : Hi, humidité volumique à la capacité au champ, profondeur racinaire, humidité volumique au point de flétrissement et $\alpha$. Les variables $\mathrm{Hi}$ et $\mathrm{Hcc}$ sont nettement plus influentes que les variables décrivant la dessiccation du sol $(\alpha, R, H P F)$. En fait ceci reflète la forme des équations où interviennent $\alpha, R$ et HPF (équation 


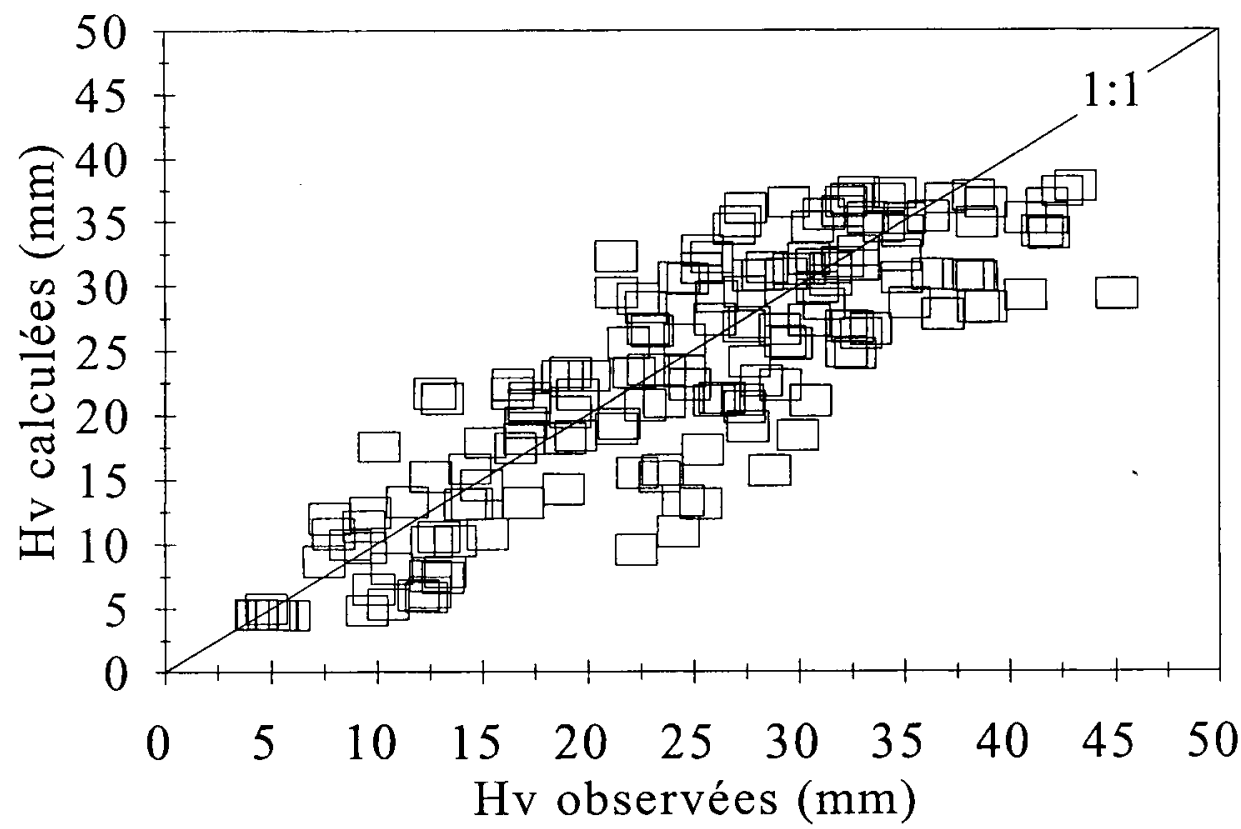

Fig 5. Humidités volumiques (Hv) calculées et observées.
[1] et [2]). L'analyse de sensibilité fait donc bien ressortir que la fiabilité de la prédiction dépend surtout de la représentativité des mesures de teneur en eau $H i$ après irrigation ainsi que de celle de la mesure de la capacité au champ.

\section{Teneurs simulées et production végétale}

Le comportement hydrique prédit dans la couche de sol $0-50 \mathrm{~cm}$ est représentatif des conditions d'alimentation en eau de la végétation. Ces conditions peuvent être évaluées en calculant le déficit moyen DM par rapport à la réserve facilement utilisable :

$$
\mathrm{DM}=\frac{\Sigma\left(H_{R F U}-H v\right)}{n} \text {, si } H v<H_{R F U}
$$

avec $H v$, l'humidité volumique calculée, $H_{R F U}$ I'humidité volumique de la réserve facilement utilisable $\left(H_{R F U}=2 R U / 3\right.$, valeur couramment utilisée dans le calcul du bilan hydrique du sol, avec $R U$ la réserve utile) et $n$ le nombre de jours où $H v<H_{R F U}$.

Le déficit moyen DM ainsi calculé est significativement corrélé ( $\left.r^{2}=0,54, n=8\right)$ avec la production de matière sèche cumulée (fig 7) pendant la période de végétation (premier cycle de végétation et regain). En revanche, aucune relation

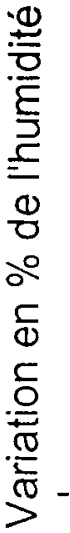

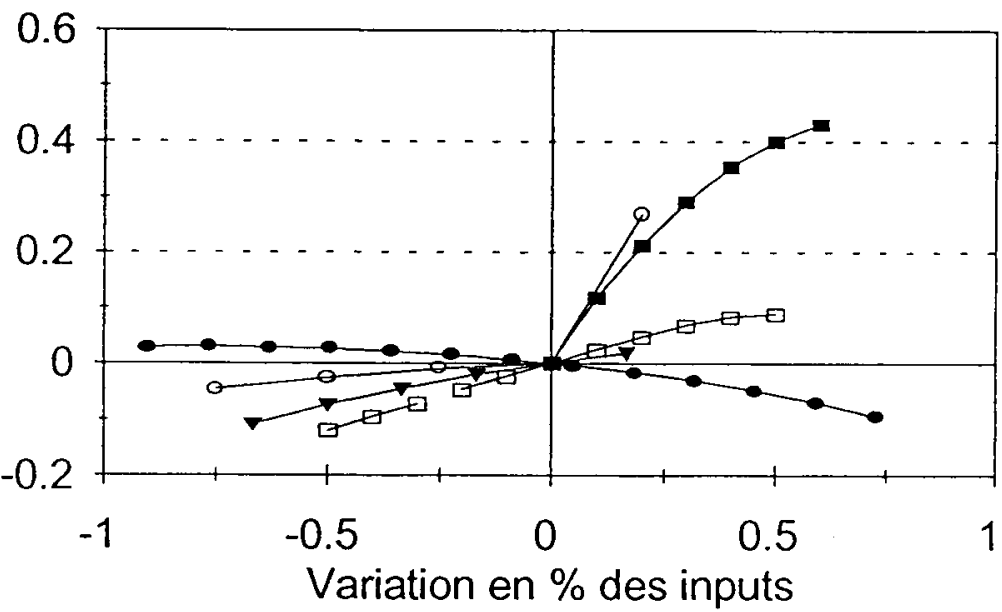

$$
\begin{array}{ll}
- \text { alpha } & \because \text { Prof Rac }-\mathrm{HCC} \\
-\mathrm{HPF} & -\mathrm{Hi}
\end{array}
$$

Fig 6. Analyse de sensibilité. Effet des variations en $\%$ des «inputs" sur la valeur de l'humidité calculée (Prof Rac : profondeur racinaire; HCC : humidité à la capacité au champ ; $\mathrm{Hi}$ : humidité initiale après irrigation ; HPF : humidité au point de flétrissement). 


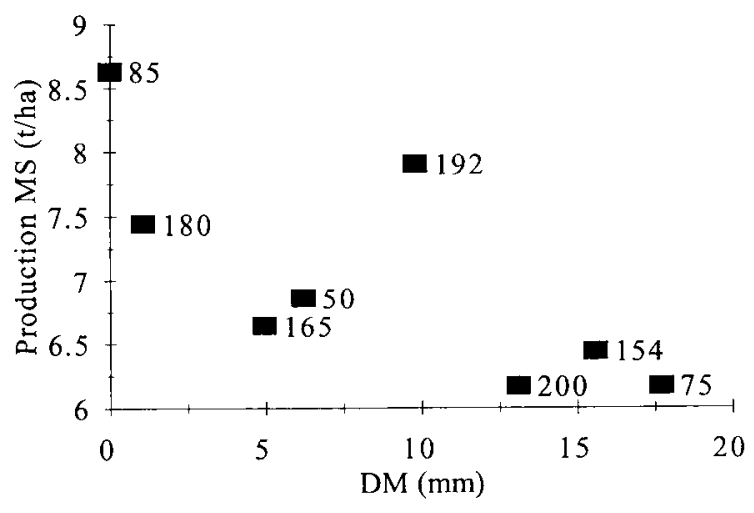

Fig 7. Influence du déficit moyen (DM) sur la production de matière sèche ( $1^{\text {er }}$ cycle et $2^{\mathrm{e}}$ cycle). Les libellés donnent la fertilisation azotée en $\mathrm{U}$ d'N

directe ne s'observe entre fertilisation azotée et rendement, à l'inverse des Alpes externes ou du Massif central, où ce sont la fertilisation azotée et le régime thermique qui sont les déterminants majeurs de la production prairiale (De Montard, 1986 ; Fleury et al, 1985).

Ceci n'exclut pas qu'il existe des interactions eau-nutrition azotée-régime thermique mais leur analyse sort du cadre de ce travail.

\section{Indicateurs de satisfaction en eau des plantes}

Les simulations réalisées montrent une bonne adéquation entre les humidités calculées par le modèle et celles observées sur le terrain. Ces calculs ont également un sens en termes de production végétale et en conséquence, nous utilisons le modèle pour déterminer le comportement hydrique dans des situations typiques de gestion des réseaux d'irrigation.

Grâce aux simulations effectuées sur la période début avril à fin août, nous calculons le nombre de jours où l'humidité volumique devient inférieure à la réserve facilement utilisable (tableau VII). On peut distinguer plusieurs types de réponses: des situations où les périodes de déficits sont inférieures à $15 \mathrm{j}$, comme par exemple dans le cas des irrigations débutant vers mi-avril ; d'autres où les périodes de déficits sont comprises entre 15 et 30 j et enfin, des cas où les périodes de déficits sont supérieures à $30 \mathrm{j}$, notamment pour les sols de la classe C1. On voit également que l'irrigation par ruissellement occasionne moins de journées de déficit que l'aspersion, surtout si cette dernière est effectuée 2 fois par mois.

\section{CONCLUSION}

La variabilité des sols et des modalités d'irrigation en val d'Aoste est bien mise en évidence par les observations et les enquêtes que nous avons effectuées. Les modalités d'irrigation sont loin d'uniformiser les régimes hydriques : après irrigation, les réserves utiles ne sont pas systématiquement reconstituées, surtout sous aspersion et dans le cas de sols ayant accumulé des déficits

Tableau VII. Nombre de jours où I'humidité volumique calculée du sol est inférieure à la réserve facilement utilisable, selon le type de sol et les modalités d'irrigation.

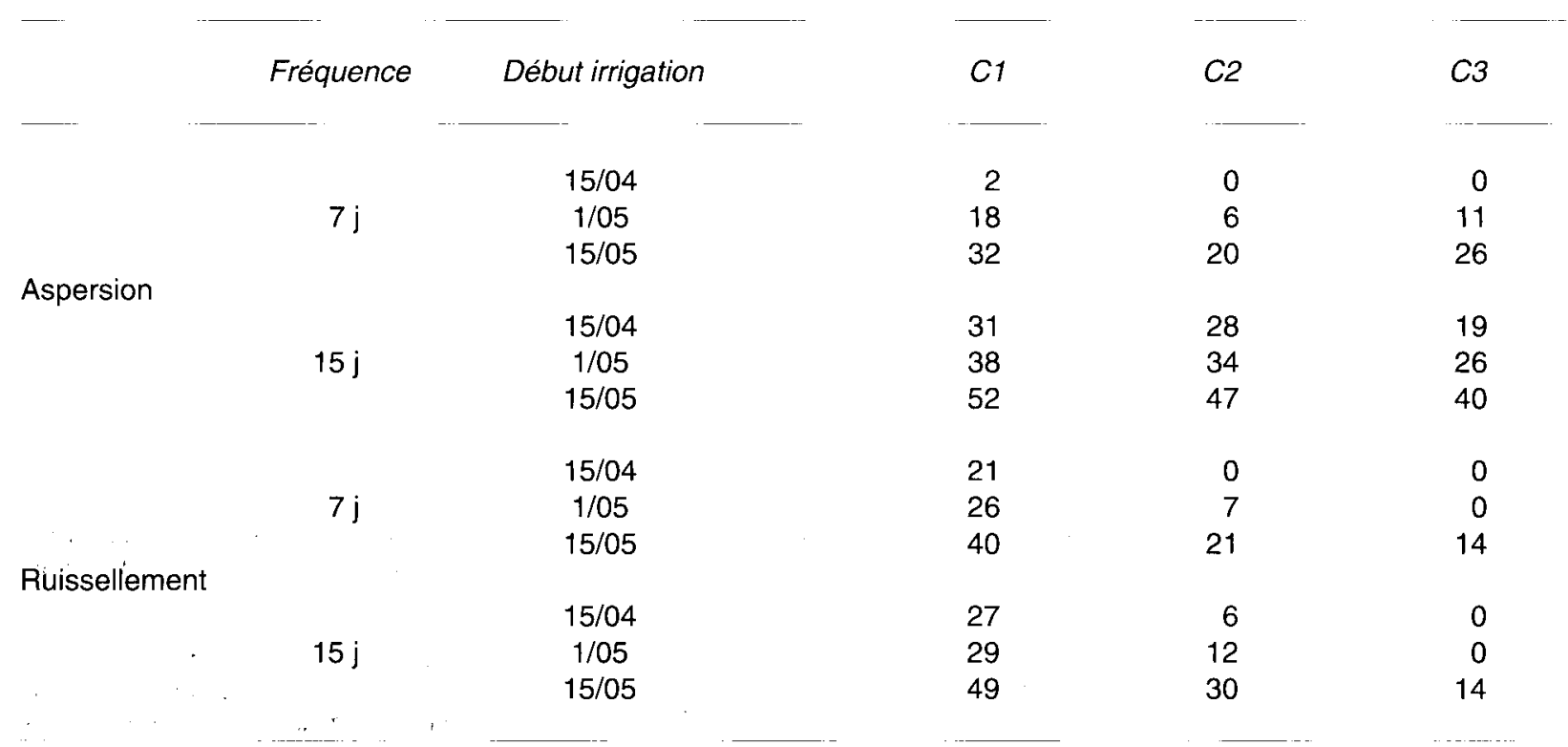


antérieurs. Enfin, comme signalé par Noitsakis et Berger (1984), le couvert végétal s'adapte aux conditions de stress hydrique des sols plus superficiels, par une meilleure prospection racinaire.

Les différents régimes hydriques peuvent être étudiés par simulation, sur la base d'un modèle de calcul des variations des teneurs en eau du sol. Après avoir adapté le module de calcul de CREAMS des variations des teneurs en eau à nos conditions expérimentales, nous avons pu vérifier la bonne adéquation des résultats des calculs à la réalité. L'ordre de grandeur des écarts est en effet comparable à celui apporté par des calculs plus élaborés (Choppart et Vauclin, 1990 ; Haith et al, 1984). Les calculs apportent de même des informations sur la disponibilité en eau pour les plantes, cohérentes avec les productions enregistrées sur des parcelles de référence.

Du fait de sa simplicité, le modèle CREAMS est bien adapté pour étudier à l'échelle régionale l'effet de variables d'entrée généralement peu précises. C'est le cas par exemple des dates et des doses d'irrigation, variables liées à des pratiques séculaires et de ce fait très difficiles à enregistrer systématiquement. C'est aussi le cas des valeurs de pluie et d'ETP qui sont très contrastées en montagne selon l'altitude, l'exposition, le relief, le régime des vents, etc (Legros et al, 1986).

Ce modèle nous a permis d'effectuer différentes simulations des variations des teneurs en eau du sol, sur la base de critères globaux tels que type de sol ou de paramètres accessibles par enquête tels que technique d'irrigation, durées probables de la saison d'irrigation, fréquences moyennes d'irrigation. Ce travail aboutit à définir le déficit hydrique des stations représentatives des conditions de production fourragère régionale. II nous permet donc de fournir un descripteur essentiel pour l'analyse des relations entre facteurs écologiques (conditions de milieu et pratiques agricoles) et états de végétation (Jeanin et al, 1991 ; Bornard et Dubost, 1992).

\section{RÉFÉRENCES}

Baize D (1988) Guide des analyses courantes en pédologie. INRA, $172 \mathrm{p}$

Bernard JF (1990) Synthèse sur l'irrigation en val $d^{\prime A}$ oste. Doc Tech TPF $n^{\circ}$ 1. IAR Aoste

Blondon G, Blanchet B, Pouly C, Flachat B, Peltier J, Martin $S$ (1987) Problèmes posés par l'irrigation en altitude. Application aux secteurs de haute
Maurienne et haute Tarentaise. Action spécifique départementale $n^{\circ} 2$, chambre Agriculture Savoie, $42 \mathrm{p}$

Boulaine J (1978) Cours d'hydropédologie. ENGREF, $195 p$

Bornard A, Dubost M (1992) Diagnostic agro-écologique de la végétation des alpages laitiers des Alpes du Nord humides : établissement et utilisation d'une typologie simplifiée. agronomie 12, 581-599

Choisnel E (1992) Le calcul du bilan hydrique du sol : options de modélisation et niveaux de complexité. Sci Sol 30, 15-31

Choppart JL, Vauclin M (1990) Water balance estimation model: field test and sensitivity analysis. Soil Sci Soc Am J 54, 1377-1384

De Montard FX (1986) Impact du climat sur la production agricole en montagne. Rapport de synthèse. Agrométéorologie des régions de moyenne montagne. In : Les colloques de I'INRA $N^{\circ} 39$, INRA, $275-279$

Deyssautier $L$ (1991) Approche du régime hydrique des prairies du val d'Aoste. Analyse in situ des modalités de remplissage des sols après irrigation. Mémoire DU univ Claude-Bernard Lyon I, $39 \mathrm{p}$

Dorioz JM, Van Oort F (1991) Approche agro-pédologique des zones pastorales sur calcshistes sédimentaires (Beaufortain-Alpes du Nord). II. Répartition des sols et des valeurs d'usage pastoral. agronomie 11, 395-409

Durand JH (1983) Les sols irrigables. PUF, Paris, $339 p$

Durand R, Legros JP (1981) Cartographie automatique de l'énergie solaire en fonction du relief. agronomie 1, 31-39

Duru M (1989) Variability of leaf area index extension rate on permanent grasslands. In: XVI International Grassland Congress, Nice, 501-502

Fleury P, Dorioz JM, Jeanin B (1985) Influence du milieu physique et des pratiques agricoles sur la végétation des prairies de fauche des hautes vallées des Alpes du Nord. Études et Recherches INRA-SAD, Versailles $n^{\circ} 3,52 p$

Gras R (1988) Physique du sol pour l'aménagement. Masson, Paris, $587 \mathrm{p}$

Haith DA, Tubbs LJ, Pickering NB (1984) Simulation of Pollution by Soil Erosion and Soil Nutrient Loss. Pudoc, Wageningen, $77 p$

Hauwy A, Folliet X, Moyroud P, Fleury P (1991) II. Utilisation de la typologie des prairies de fauche des Alpes du Nord. Fourrages 128, 397-406

Henin S, Gras R, Monnier G (1969) Le profil cultural. L'état physique du sol et ses conséquences agronomiques. Masson, Paris, $332 \mathrm{p}$

ITCF (1993) Gérer l'irrigation en grande culture. ITCF, Boigneville, $56 p$

Janin B (1967) Le val d'Aoste. Traditions et renouveau. Musumecci, $684 \mathrm{p}$

Jeanin B, Fleury P, Dorioz JM (1991) Typologie régionale des prairies permanentes fondée sur leur apti- 
tude à remplir des fonctions. Fourrages 128, $3377-$ 422

Kauark Leite LA (1990) Réflexions sur l'utilité des modèles mathématiques dans la gestion de la pollution diffuse d'origine agricole. Thèse doc ing, École nationale ponts-et-chaussées, $341 \mathrm{p}$

Knisel WG (1980) CREAMS: A field-scale model for chemical runoff and erosion from agricultural management systems. US Department of Agri Conservation Research, Report N ${ }^{\circ} 26,640 p$

Legros JP (1984) Introduction à l'étude de la simulation de l'évolution granulométrique du sol. Présentation d'un modèle informatique. Sci Sol 1, 51-63

Legros JP, Antonioletti R, Genre-Grandpierre (1986) Évaluation de l'énergie solaire en zone montagne. Agrométéorologie des régions de moyenne montagne. In : Les colloques de I'INRA N $N^{\circ}$ 39, INRA, 8793

Legros JP (1986) Cartographie des paysages pédologiques dans les alpes humides. Exemple du Chablais. Agrométéorologie des régions de moyenne montagne. In : Les colloques de I'INRA $N^{\circ} 39$, INRA, 119-127

Lyszczarz R (1989) Influence de la fertilisation sur la répartition de la biomasse végétale en prairie temporaire. In : International Grassland Congress, Nice, 51-52

Meddahi E, Mallants D, Feyen J, Vereecken H (1993) Modélisation de l'évolution de la salinité dans la zone racinaire. Sci Sol 31, 47-58

Noitsakis B, Berger A (1984) Relations hydriques chez Dactylis glomerata et Dichantium ischaemum cultivés sous 2 régimes hydriques contrastés. Acta Oecologica Oecol Plant 5, 75-88

Ozenda P (1981) Carte de la végétation de la France au $200000^{e}$. Végétation des Alpes sud-occidentales. CNRS, $258 \mathrm{p}$

Puech J (1969) Étude expérimentale de la circulation de l'eau non saturante de différents sols vers une zone d'absorption. Ann Agron 20 (3), 245-261
Rochas O (1991) Suivi des cinétiques de vidange après irrigation des principaux sols de prairies en vallée d'Aoste. IAR, Aoste, $41 \mathrm{p}$

Roumet JP, Fleury P, Pauthenet Y, Neyroz A (1992) Variabilité régionale et déterminisme des pratiques parcellaires sur prairies permanentes en vallée d'Aoste. In : Annales de l'Institut agricole régional, tome I, Aoste, 83-97

Tardieu F (1987) État structural, enracinement et alimentation hydrique du maïs. III. Disponibilité des réserves en eau du sol. agronomie 7 (4), 279-288

Toth S (1989) The above-ground and root production of grass on different soils. In : XVI International Grassland Congress, Nice, 11-12

Trévisan D, Pauthenet $Y$, Roumet JP (1992) Les sols de prairie en val d'Aoste : essai de classification et étude du comportement de leur réservoir hydrique après irrigation. In : Annales Institut agricole régional, tome I, Aoste, 99-112

Vauclin M (1990) Modélisation des transferts dans les sols non saturés : approche déterministe ou stochastique? In: Nitrates eau agriculture, INRA, 169181

Voltz M, Rambal S (1987) Évaluation de CORNGRO : un modèle de simulation des transferts hydriques et de la croissance d'une culture de maïs. agronomie 7, 727-738

Williams JR, Haan RW (1978) Optimal operation of large agricultural watersheds with water quality constraints. Texas Water Resources Institut, Texas A \& M University, Technical report $N^{\circ} 96$, $152 \mathrm{p}$

Williams JR, Smith RE, Nowlin JD, Nicks AD (1980) Hydrology in CREAMS: A field-scale model for chemical runoff and erosion from agricultural management systems. US Department of Agri Conservation, Research Report N ${ }^{\circ} 26,165-192$

West CP, Beyrouty CA, Gbur EE (1989) Responses of root and shoot growth of Cynodon Dactylon to cutting frequency and growth regulator. In : XVI International Grassland Congress, Nice, 529-530 